\title{
Design and Development of Software Configuration Management Tool to Support Process Performance Monitoring and Analysis
}

\author{
Alan Cline ${ }^{1}$, Eun-Pyo Lee ${ }^{2}$, and Byong-Gul Lee ${ }^{2}$ \\ ${ }^{1}$ Ohio State University, Department of Computer Science and Engineering, \\ Columbus, Ohio, USA \\ acline@carolla.com \\ ${ }^{2}$ Seoul Women's University, Department of Computer Science, \\ Seoul, Korea \\ eun-pyo@hanmail.net, byonglaswu.ac.kr
}

\begin{abstract}
Most SCM tools underestimate the potential ability of monitoring and reporting the performance of various software process activities, and delegate implementation of such capabilities to other CASE tools. This paper discusses how SCM tool can be extended and implemented to provide valuable SCM information (e.g., metric data) in monitoring the performance of various process areas. With the extended SCM tool capability, stakeholders can measure, analyze, and report the performance of process activities even without using the expensive CASE tools.
\end{abstract}

Keywords: Software Configuration Management, Process Metric.

\section{Introduction}

Software Configuration Management (SCM) is a key discipline for development and maintenance of large and complex software systems [1], [2]. Many researches and studies show that SCM is the most basic management activity for establishing and maintaining the integrity of software products produced throughout the software life cycle. The activities of SCM include identifying configuration items/units, controlling changes, maintaining the integrity and the traceability of the configuration item, and auditing and reporting of configuration management status and result.

The existing configuration management tools support some or the combination of these activities with the help of functions including change control, version control, work space management, and build/release control [3]. However, most SCM tools underestimate the benefits of using the SCM metric capability in monitoring and measuring of other process areas and have their implementation depend on other CASE tools such as project management tool or spreadsheet software [4]. We believe that the SCM tool capability can be extend to provide some valuable services and information for monitoring and measuring the performance of various process activities, such as project management, requirement management, or software quality assurance. For example, to monitor the stability of requirements $\left(S_{R}\right)$ during 
requirement management, some of the primitive metric data from the change control function can be utilized as follows:

$$
S_{R}=N_{C R} /\left(\sum_{i} \text { Initial Requirements }_{i} \cdot T_{\mathrm{i}}\right) .
$$

where $\mathrm{N}_{C R}$ represents the total number of change request on a requirement and $T_{i}$ is the time interval between the initial check-in and the final release of a requirement.

Current SCM tools rarely provide the mechanism for incorporating the management and utilization of SCM metrics into other process areas. They usually define only a few set of primitive SCM metrics and let the thirsty user utilize them on a need basis. Besides, the current tools have no way of monitoring and measuring the performance for a newly defined process or out of the defined scope. For example, a user might be interested in measuring the effectiveness of the training plan prepared for an organization or a senior manager might want to create a new process role with a new responsibility and to see how the jobs get done. In this situation, the user is prone to purchase a tool fit to that purpose.

This paper describes the design and development of SCM tool which can provide the measurement, analysis, and reporting of the process performance in the broader process areas. The remainder of this paper is organized as follows. Section 2 gives the related works by comparing the exiting SCM tool capability based on metric utilization. Section 3 and 4 describe the design and usage of our SCM tool in supporting of metric capability. Finally, the conclusion and future works appears in Section 5 .

\section{Current Configuration Management Tools}

The features of the current SCM tools are limited to the support of only a few areas such as requirement management or project tracking and oversight. Covering the entire software process activities then costs for purchasing the expert tools.

IBM's ClearQuest provides a workflow management capability and enforce the process monitoring. However, for this service to be useful, it needs to integrate with a tool, Portfolio Manager, for the profiling of process information from the extern [5]. Telelogic's Synergy, integrated with Dashboard, intends to support a decision making of project manager by automating data collection, analysis, and reporting of measurement data. However, the tool only focuses on automating the requirement management process data [6]. Borland's StarTeam tends to offer a comprehensive solution that includes integrated requirements management, change management, defect tracking, and project and task management [7]. However, its primary usage is limited to the SCM only and depends on external CASE tools to utilize the SCM information in other process activities. Continuus/CM toolset also cannot be used for the complicated process environment due to its lack of process related information [8], [9]. The study of [10] supports our view by stating that the current SCM tools have: 1) No support with the planning function; 2) No sufficient support with process related functions; 3) No support with the report and audit mechanisms; 4) No support with measurement. 


\section{Development of Configuration Management Process Tool}

To lessen the problem of existing SCM tools, we propose a Configuration Management Process Tool (CMPT) by implementing features such that the software project team can monitor and analyze their process performance by utilizing the SCM metrics for each process area. CMPT is implemented in JAVA and runs on a singleuser workstation connected to a remote shared CVS server.

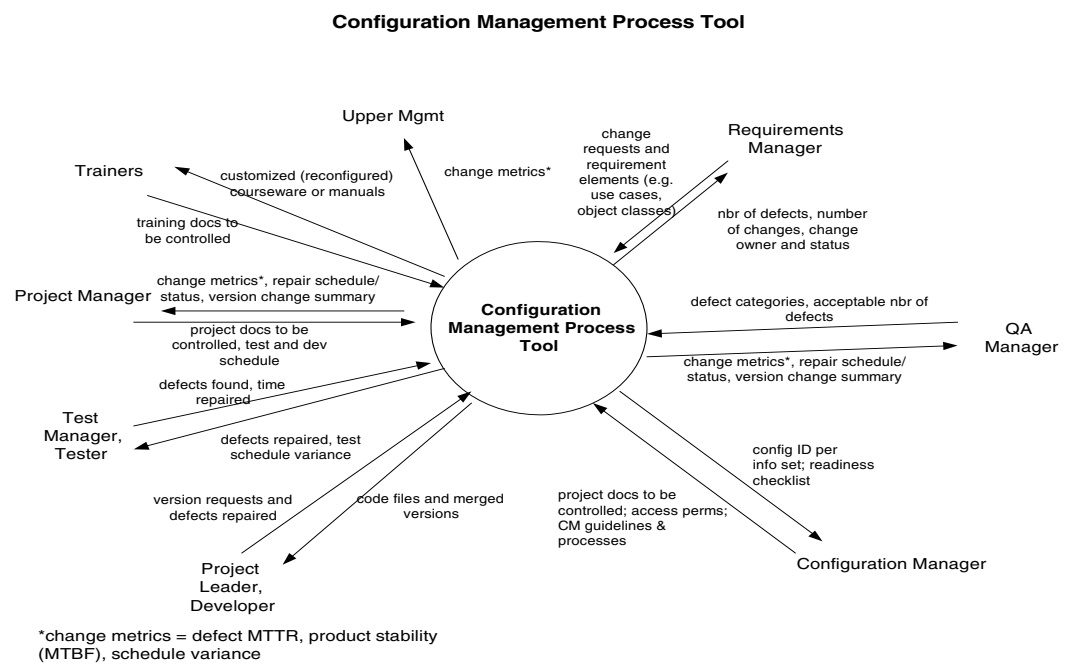

Fig. 1. Context Diagram of the CMPT

Figure 1 illustrates the scope of CMPT and data flow that comes in and out of a system. The requirements manager, for instance, inputs the requirement artifacts for controlling and monitoring of use cases and object model classes. CMPT can deliver to requirement manager the status reports containing the number of defects, the number of changes and change requests, the change owner and date, and the status of change requests.

\subsection{Change Control Process}

Among the many features of CMPT, the change control provides a base playground for monitoring and collecting the various process data. The state flow diagram in Figure 2 represents the invariants, pre-conditions, and post-conditions of change control process.

The change control flows are divided into two modes, free text module and executable module. The free text module elements (e.g., Proposal, Charter, Requirement Specification, Design, Test Plan, Training Plan, and etc.) can have several states: REQUEST PROPOSED, APPROVED, COMPLETE, and some intermediate states. Executable modules (e.g., Change Requests, Defects, Code, Test Cases, and Use 
Cases) go through seven states: REQUEST PROPOSED, APPROVED, ASSIGNED, IMPLEMENTED, TEST READY, TESTED and COMPLETE states. CMPT is designed to monitor and collect the status information for each of these states to provide more accurate and affluent metrics for other process areas.

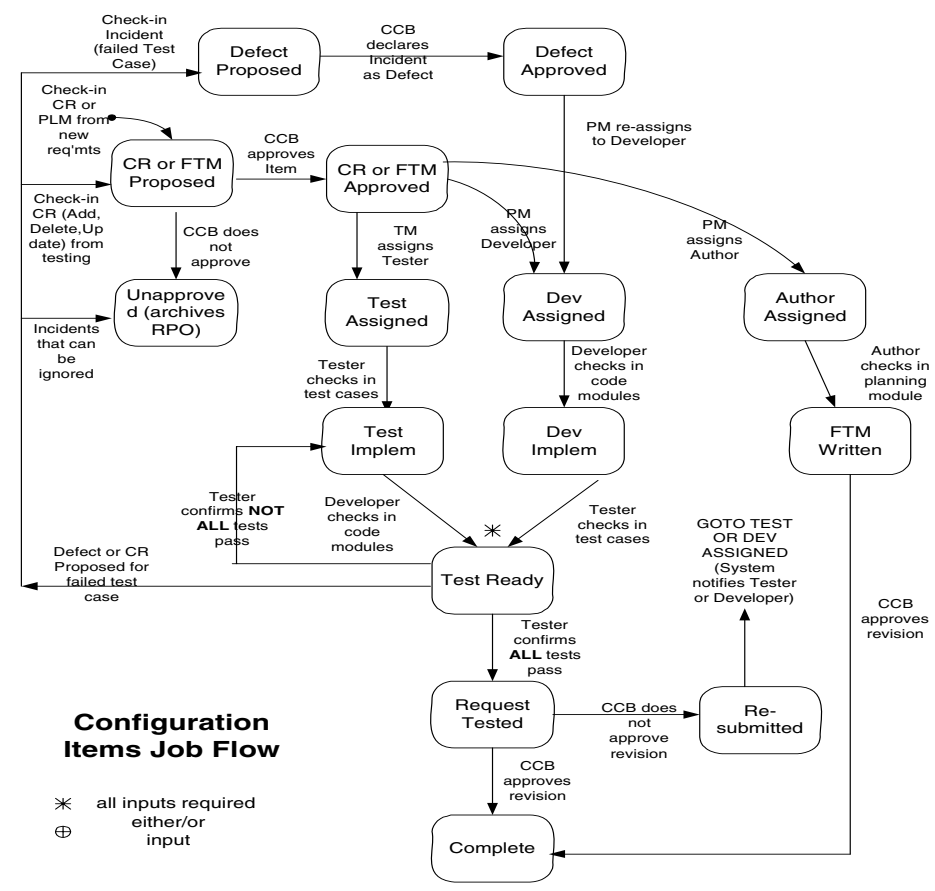

Fig. 2. Flow Items of the SCM Change Process

\subsection{User Profiles and Transaction Permissions}

CMPT facilitates with an access control mechanism for it can be utilized by different participants in various process areas. Table 1 shows each user's transaction CMPT facilitates. The Project Manager will input project documents to be controlled, and the test and development schedule. The Project Manager then want change metrics, repair schedules, development and change statuses, and version change summaries for the product from CMPT. The Requirements Manager may input requirement elements for control and monitoring and want CMPT to deliver reports containing the number of defects, number of changes and change requests, the change owner and date, and status of change requests and repairs. The Test Manager and Tester may want CMPT to generate defect repair reports (change metrics) and test schedule variance. Both Developers and Project Leaders may want CMPT to produce merged product versions, code files, traceable version links, and change reports. CMPT can provide Configuration Manager with version or configuration ID and configuration status 
profiles to help coordinating the various SCM activities. CMPT also provides the audit mechanism to enforce the CM guidelines and policies as planned. For the QA Manager, CMPT provides various QA metrics for each process area under the control of SCM.

Table 1. Example of Each User's Transaction Permissions

\begin{tabular}{|l|l|l|l|l|l|l|l|l|l|}
\hline User & PM & RM & QM & CM & TM & $\begin{array}{l}\text { Proj. } \\
\text { Leader/ } \\
\text { Developer }\end{array}$ & Tester & Trainer & $\begin{array}{l}\text { General } \\
\text { Users }\end{array}$ \\
\hline $\begin{array}{l}\text { Get metric } \\
\text { report }\end{array}$ & $\sqrt{ }$ & $\sqrt{ }$ & $\sqrt{ }$ & $\sqrt{ }$ & $\sqrt{ }$ & $\sqrt{ }$ & $\sqrt{ }$ & & \\
\hline $\begin{array}{l}\text { Check out } \\
\text { item }\end{array}$ & $\sqrt{ }$ & $\sqrt{ }$ & $\sqrt{ }$ & $\sqrt{ }$ & $\sqrt{ }$ & $\sqrt{ }$ & $\sqrt{ }$ & & $\sqrt{ }$ \\
\hline $\begin{array}{l}\text { Change } \\
\text { request }\end{array}$ & $\sqrt{ }$ & $\sqrt{ }$ & $\sqrt{ }$ & $\sqrt{ }$ & $\sqrt{ }$ & $\sqrt{ }$ & $\sqrt{ }$ & $\sqrt{ }$ & \\
\hline $\begin{array}{l}\text { Approve } \\
\text { change } \\
\text { request }\end{array}$ & $\sqrt{ }$ & $\sqrt{ }$ & $\sqrt{ }$ & $\sqrt{ }$ & $\sqrt{ }$ & & & & \\
\hline
\end{tabular}

\subsection{Process Metric}

The key to the process metrics analysis is to analyze the actual effort, schedule, deviation of cost and plan, and defects during the project [11], [12]. Table 2 shows a sample of such metrics provided in [11]. In CMPT, these metrics can be calculated from Cartesian product of the fine grained SCM events (e.g., check-in, release, checkout, change request, change, change completion, and etc.) and scale measurements (e.g., number of events, frequency of events, time interval, average time, and etc.).

Table 2. SCM Process Metrics

\begin{tabular}{|l|l|}
\hline \multicolumn{1}{|c|}{ Metric } & \multicolumn{1}{c|}{ Equation } \\
\hline Schedule variance & (Actual duration-Planned duration)/Planned duration \\
\hline Effort variance & (Actual effort-Planned effort)/Planned effort \\
\hline Size variance & (Actual size-Planned size)/Planned size \\
\hline Change stability & Number of change request/Total number of baseline items \\
\hline Change density & $\begin{array}{l}\text { Number of changes for each baseline/Time span between check } \\
\text { in and out }\end{array}$ \\
\hline Residual change density & Number of changes completed / Number of changes requested \\
\hline $\begin{array}{l}\text { Change distribution in } \\
\text { each development phase }\end{array}$ & Number of changes in each phase/Total number of changes \\
\hline
\end{tabular}

\section{Work Scenarios of CMPT}

This section describes how CMPT can be utilized in producing metrics for monitoring other process activities. Figure 3 shows that CMPT facilitates a set of transactions and access permission associated with user's role. A user can define and customize various roles and transactions according to their process condition and environment. 

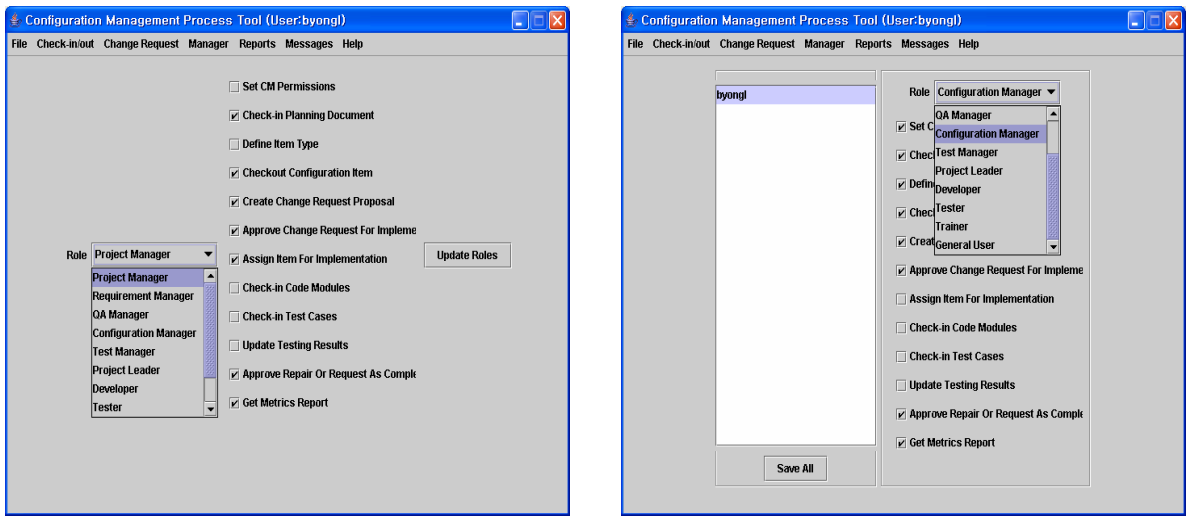

Fig. 3. Setting SCM Permissions

Figure 4 and 5 show how user can retrieve or store a configuration item. For checking out a configuration item (Figure 4), CMPT facilitates an exclusive locking and shows a lock icon for the items that already have been checked out. For checking in (Figure 5), a user can check in either free-text documents or executable elements. In CMPT, both the check-in and the check-out event are combined with the scale measurement (e.g., time, frequency, number and etc.) to produce the finer grained SCM metrics, such as number of check-in or time of check-out.

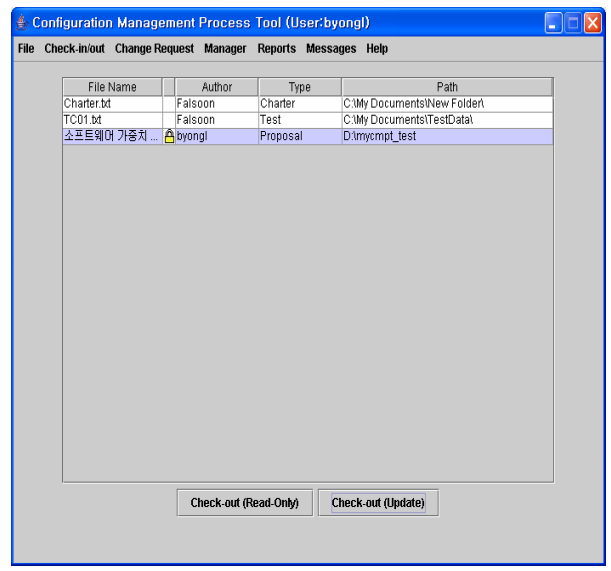

Fig. 4. Check-out of Configuration Item

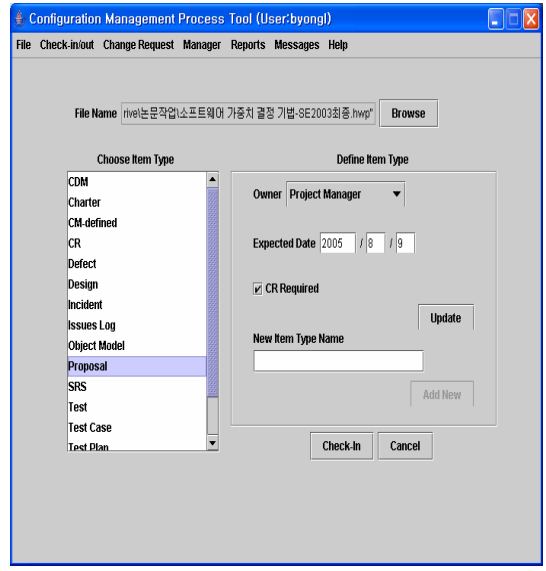

Fig. 5. Check-in of Configuration Item

Any project team member can propose a change request for adding new item(s), and replacing and deleting any existing item(s) with reasons and expected completion time (Figure 6). All element types of new items are pre-defined by the Configuration Manager. Once a Change Request (CR) is proposed, the CCB reviews the CR or defect for approval or disapproval. If CR or defect is approved, the Project Manager and Test Manager assign the request to developers and testers for implementation. 


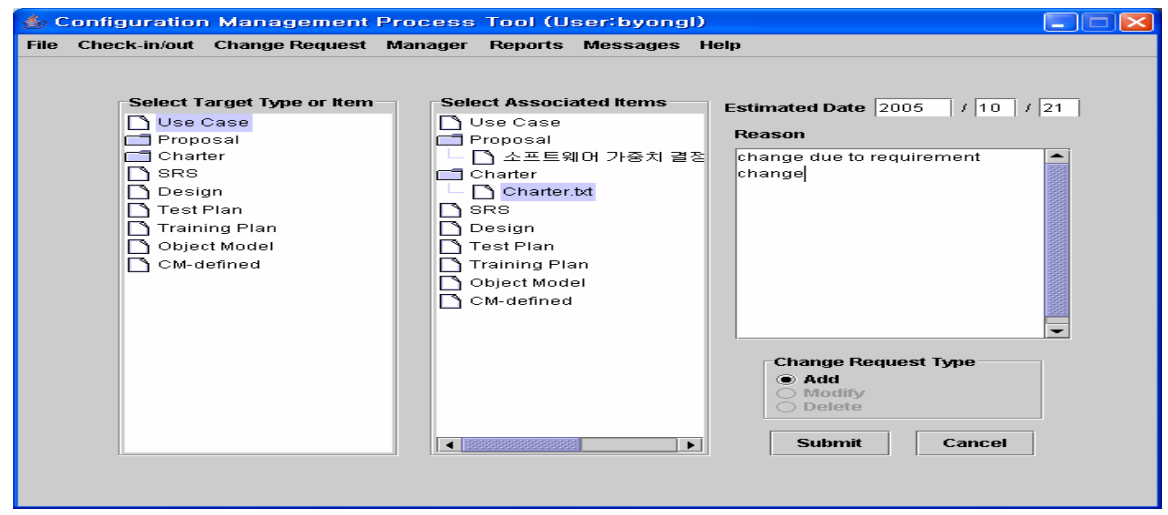

Fig. 6. Create a Change Request Proposal

User can get a metrics report for a selected configuration item with a graph (Figure 7). The graph view can provide a summary of various process performances. Users can select a report type from predefined types such as configuration status, history, tracking, or release report. The scale can be chosen from number of events, frequency of events, time interval, and average time, and the metrics can be chosen from check-in, release, check-out, change request, change, and change completion of configuration items. Figure 7 indicates the frequency of change request for each use case. In this case, use case no. 2 shows the highest frequency of change requests.

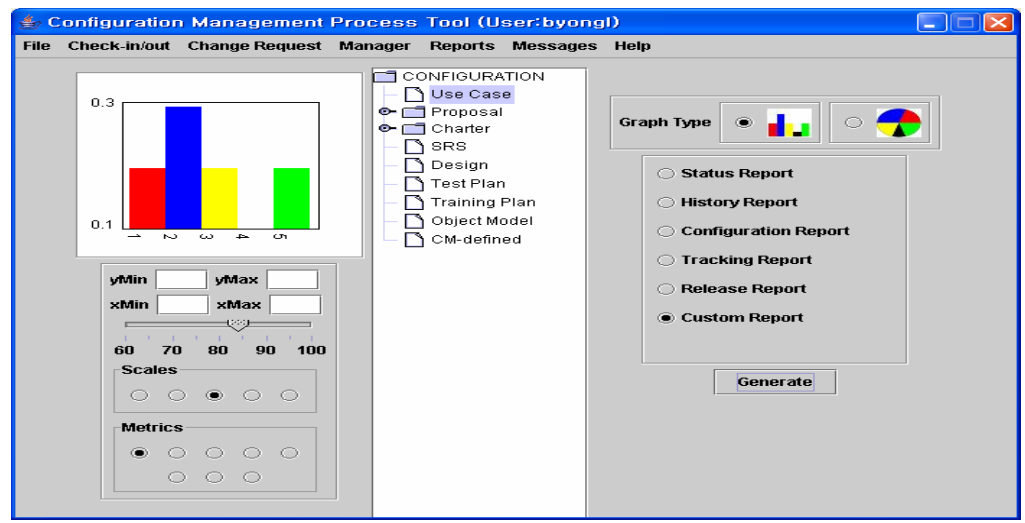

Fig. 7. Get Metrics Report

\section{Conclusion and Future Work}

SCM's capability has to extend to provide some valuable services to monitor other process or management activities, such as project management or software quality assurance. Current SCM tools rarely provide such a monitoring capability nor provide fine-grained data useful enough. This paper described the design and development of 
an SCM tool which can provide the measurement, analysis and reporting capability for monitoring other process performance without using expensive CASE tools. The proposed CMPT tool can:

1. define and customize the access/role control and associated transaction selection,

2. define and customize the process work flow, and

3. utilize the work flow status information and metrics to provide process performance information.

Currently, the CMPT's process customization capability only applies to change control. For future work, the customization scheme should be extended and enhanced to adopt the various process characteristics and project environments. More studies should also be focused on the reporting, including graphing, extraction, and translation of process metric data.

Acknowledgments. This research was supported by the MIC (Ministry of Information and Communication), Korea, under the ITRC (Information Technology Research Center) support program supervised by the IITA (Institute of Information Technology Advancement) (IITA-2006-(C1090-0603-0032)).

\section{References}

1. S. Dart: "Concepts in Configuration Management Systems," Proc. Third Int'l Software Configuration Management Workshop (1991) 1-18

2. D. Whitgift: Methods and Tools for Software Configuration Management, John Wiley and Sons (1991)

3. A. Midha: "Software Configuration Management for the 21st Century," TR 2(1), Bell Labs Technical (1997)

4. Alexis Leon: A guide to software configuration management, Artech House (2000)

5. IBM Rational: ClearCase, http://www-306.ibm.com/software/awdtools/changemgmt/ (2006)

6. Peter Baxter and Dominic Tavassoli: "Management Dashboards and Requirement Management," White Paper, Telelogic (2006)

7. Borland: Starteam, http://www.borland.com/us/products/starteam/index.html.

8. Continuus Software/CM: Introduction to Continuus/CM, Continuus Software Corporation (1999)

9. Merant :PVCS, http://www.merant.com/products/pvcs/

10. Fei Wang, Aihua Ren: "A Configuration Management Supporting System Based on CMMI," Proceedings of the First International Multi-Symposiums on Computer and Computational Sciences, IEEE CS (2006)

11. R. Xu, Y. Xue, P. Nie, Y. Zhang, D. Li: "Research on CMMI-based Software Process Metrics," Proceedings of the First International Multi-Symposiums on Computer and Computational Sciences, IEEE CS (2006)

12. F. Chirinos and J. Boegh: "Characterizing a data model for software measurement," Journal of Systems and Software, v. (74), Issue 2 (2005) 207-226 